\title{
Sustainable Antibiofouling Properties of Thin Film Composite Forward Osmosis Membrane with Rechargeable Silver Nanoparticles Loading
}

\author{
Zhongyun Liu and Yunxia $\mathrm{Hu}^{*}$
}

CAS Key Laboratory of Coastal Environmental Processes and Ecological Remediation; Research Center for Coastal Environmental Engineering and Technology of Shandong Province; Yantai Institute of Coastal Zone Research, Chinese Academy of Sciences, Yantai, Shandong Province 264003, People's Republic of China

\section{Supporting Information}

ABSTRACT: Microbial attachment and biofilm formation on filtration membrane can greatly compromise its flux and separation efficiency. Here, a simple and facile approach has been developed to in situ generate silver nanoparticles (Ag NPs) on the thin film composite forward osmosis (TFC FO) membrane for sustainable antibiofouling performances. Mussel-inspired dopamine chemistry was applied to grow polydopamine coating on both surfaces of FO membranes, followed by the generation of Ag NPs upon a simple dip coating in silver nitrate aqueous solution. Furthermore, the Ag NPs deposited membranes had a long-term silver release profile with rechargability for multiple times upon their depletion, and exhibited strong sustainable bactericidal efficacy against Gram-negative bacteria and Grampositive bacteria. The Ag NPs had a controllable effect on the membrane performances including the water flux and reverse salt flux in the FO test mode. Our practicable antibacterial strategy may apply to other types of filtration membranes with diverse material surfaces and may pave a new way to achieve the sustainable membrane antibiofouling performance on a large scale.

KEYWORDS: thin film composite, forward osmosis, membrane biofouling, silver nanoparticles, polydopamine, antibacterial properties, bactericidal efficacy

\section{INTRODUCTION}

Membrane-based seawater desalination and wastewater treatment processes have become one of the most effective and efficient technologies to produce high quality drinking water, thus relieving stress from drinking water scarcity locally and globally. ${ }^{1,2}$ Forward osmosis (FO), as a state-of-the-art membrane technology, has been gaining increasing attention in many applications including desalination, water purification, ${ }^{3,4}$ wastewater reuse, ${ }^{5}$ landfill leachate treatment, ${ }^{6}$ food processing, ${ }^{7}$ and sustainable power generation..$^{8-10}$ Membrane biofouling, caused by adsorption, deposition, and growth of microorganisms on the membrane surface, has strong negative effects on the operational sustainability and the cost-efficiency of membrane process, ${ }^{11,12}$ yet no simple cleaning method can be applied to remove biofilm efficiently and to recover the membrane properties successfully. ${ }^{13,14}$

Silver nanoparticles (Ag NPs) are widely used as most popular disinfectants to mitigate membrane biofouling because of their excellent broad-spectrum antimicrobial properties and low toxicity toward mammalian cells. ${ }^{15-18}$ Both in situ and ex situ methods have been developed to incorporating Ag NPs into the membrane surface. In the first approach, with the aid of reducing agents, the synthesis of Ag NPs occurred on the

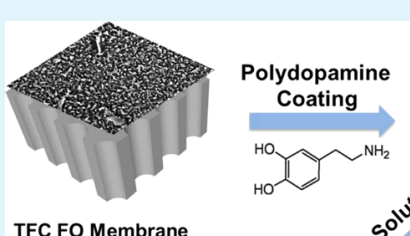

TFC FO Membrane

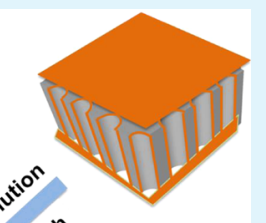

$2^{n}$

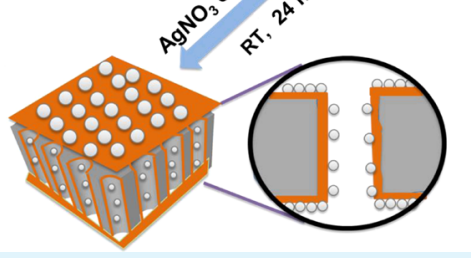

membrane surface sequestering silver ions. ${ }^{19,20}$ For example, the negatively charged carboxylic groups sequestered $\mathrm{Ag}^{+}$on the polyamide surface of reverse osmosis (RO) membrane, and the silver ions were reduced by sodium borohydride $\left(\mathrm{NaHB}_{4}\right)$ to form Ag nanoparticles. ${ }^{21}$ However, the Ag loading amount was very low, and the formed Ag NPs were loosely bound on the surface and were easily washed out. The ex situ method generally involves two steps including the synthesis of Ag NPs first and their immobilization on the membrane surface, requiring either Ag NPs or membrane surface being functionalized to promote their strong bonding. ${ }^{22}$ For example, the thiol-rich surface of TFC membrane was engineered first, and then bound with the freshly synthesized Ag NPs via the Ag-S chemical bonding. ${ }^{23,24}$ Above all, all of these approaches require the membrane surface having certain functionalities to immobilize Ag NPs, and they are not applicable to most of other different membranes.

Mussel-inspired dopamine chemistry has opened a new route to modify all types of inorganic and organic substrates with

Received: June 6, 2016

Accepted: July 28, 2016

Published: July 28, 2016 
controllable film thickness and durable stability. ${ }^{25,26}$ Polydopamine (PDA) has stimulated extensive research in the membrane surface functionalization for antifouling purpose. $^{27-29}$ Interestingly, PDA has been found to reduce $\mathrm{Ag}^{+}$ ions to $\mathrm{Ag}$ and to synthesize $\mathrm{Ag}$ NPs in situ on different substrates without any other reducing agent. ${ }^{30-33}$ Moreover, the $\mathrm{O}$ - and $\mathrm{N}$-sites of PDA can serve as anchors for the Ag NPs to enhance the durability of Ag NPs on the surfaces via metal coordination. $^{25}$ Therefore, PDA chemistry may pave a simple, yet universal way to immobilize Ag NPs on various membrane surfaces for antibiofouling purpose. However, to date, a few studies applied the PDA chemistry only on polysulfone ultrafiltration membranes to generate Ag NPs achieving antimicrobial properties. ${ }^{34,35}$ No work has been done to investigate the rechargability of Ag NPs on the PDA modified surfaces and their sustainable antimicrobial properties. More importantly, the generation of Ag NPs through PDA chemistry has not been tested on the most common well-used thin film composite (TFC) membranes with a polyamide layer. The influences of $\mathrm{Ag}$ NPs deposition on the membrane performances are still not well-understood including water flux and salt rejection.

Herein, we are the first to generate in situ Ag NPs simultaneously on both surfaces of TFC FO membranes including polysulfone support layer and polyamide active layer based on the PDA chemistry, and to investigate the effects of Ag NPs on the morphology and roughness of polyamide layer along with the water flux and salt rejection of FO membranes. Furthermore, for the first time, the rechargability of Ag NPs was demonstrated on the membranes through the PDA chemistry upon their depletion, and thus the long-term antibacterial properties of the membranes were also investigated. Our results shed new light on the development of a simple, universal, and applicable approach for constructing long lasting biofouling-resistant membrane surfaces.

\section{MATERIALS AND METHODS}

2.1. Materials and Chemicals. The commercial thin film composite forward osmosis (TFC FO) membranes were purchased from Hydration Technology Innovations (HTI, Albany, OR), and the membrane coupons were wetted through immersion in $25 \%$ isopropyl alcohol solution for $30 \mathrm{~min}$, followed by thorough rinse with deionized (DI) water and stored at $4{ }^{\circ} \mathrm{C}$ before test or further modification. Dopamine was purchased from Sigma-Aldrich (St. Louis, MO, USA). Silver nitrate and $68 \%$ nitric acid were obtained from Sinopharm Chemical Reagent Beijing Co., Ltd., China and used as received. $1 \mathrm{M}$ Tris-HCl buffer $(\mathrm{pH}=9.0)$ was obtained from Beijing Solarbio Science \& Technology Co., Ltd., China. Escherichia coli (E. coli, DH5 $\alpha$ ) was purchased from Beijing Dingguo Changsheng Biotechnology Co., Ltd., China. Staphylococcus aureus (S. aureus, CICC 10201) was obtained from China Center of Industrial Culture Collection.

2.2. Surface Modification and in Situ Formation of Ag NPs on TFC FO Membrane. The prewetted TFC FO membrane coupons were soaked in $100 \mathrm{~mL}$ of a $2 \mathrm{mg} / \mathrm{mL}$ fresh prepared dopamine solution at $\mathrm{pH} 8.510 \mathrm{mM}$ Tris- $\mathrm{HCl}$ buffer in a glass pan and kept agitated in a shaking incubator for $6 \mathrm{~h}$ at room temperature to allow self-polymerization of dopamine under air and formation of PDA. ${ }^{36}$ Following that, the membrane coupons were thoroughly rinsed with deionized water three times to obtain the PDA coated TFC FO membrane. To generate Ag-NPs on the FO membrane, the PDA coated membrane coupons were simply incubated in $100 \mathrm{~mL}$ of a 50 $\mathrm{mM}$ silver nitrate aqueous solution for $24 \mathrm{~h}$ at room temperature, and rinsed three times with DI water to remove unbound silver ions. The membrane coupons were air-dried at room temperature and kept in the dark for further characterization.
2.3. Membrane Characterization. Scanning electron microscopy (SEM, S-4800, Hitachi) was used to observe the distribution and morphologies of the Ag NPs on the membrane surfaces and cross sections. Cross-sectional samples were prepared by snapping the wet membranes in liquid nitrogen. An EMITECH SC7620 sputter coater was utilized to coat all samples with $10 \mathrm{~nm}$ thick platinum $(\mathrm{Pt})$ for 100 $s$ before SEM and energy-dispersive X-ray (EDX) observation. EDX analyses were conducted to detect $\mathrm{Ag}$ on the membranes with $15.0 \mathrm{kV}$ accelerating voltage. The static contact angles of modified membranes were detected using an optical instrument (OCA20, Data Physics, Germany). Using the sessile drop method, a $2 \mu \mathrm{L}$ of water droplet was placed on the air-dried membrane surfaces and photographed with a digital camera after $5 \mathrm{~s}$. For each data point, ten measurements were performed on each sample and three individual specimens were tested for parallel experiments. Errors were given as the standard deviation. $\mathrm{X}$-ray photoelectron spectroscopy (XPS, Thermo Esca Lab 250Xi, Thermo Fisher Scientific, USA) was employed to detect the elemental composition of the membrane surface with monochromatic $\mathrm{Al} \mathrm{K \alpha} \mathrm{X}$ ray source $(h v=1486.6 \mathrm{eV})$. The photoelectron takeoff angle was $60^{\circ}$ and dwell time was $0.1 \mathrm{~s}$ with one time scanning. The full range data was collected by survey scan $(200-800 \mathrm{eV}$, step size: $1 \mathrm{eV})$. The $\mathrm{Ag}$ NPs loadings of the membranes were determined by soaking the membrane samples in a $3.5 \% \mathrm{HNO}_{3}$ solution for $48 \mathrm{~h}$ and measuring the dissolved $\mathrm{Ag}$ concentrations by an inductively coupled plasma mass spectrometry (ICP-MS, ELAN DRC II, PerkinElmer, Ltd., Hong Kong), as the published method. ${ }^{21}$ Atomic force microscopy (AFM, Multi-Mode 8, Veeco, US) measurements were performed with tapping mode in air using TESP tips (Sharp silicon probe, $42 \mathrm{~N} / \mathrm{m}$, $320 \mathrm{kHz}$, tip radius $8 \mathrm{~nm}$, no coating, Bruker, USA), and Nanoscope Analysis Software was used to calculate the various roughness parameters.

2.4. Antimicrobial Activity of TFC FO Membranes. The colony forming unit (CFU) counting method ${ }^{21,37,38}$ was utilized to assess the antimicrobial properties of the membranes using two model bacteria: E. coli and S. aureus. An overnight culture of bacteria grown in LuriaBertani (LB, $1 \mathrm{~mL}$ ) broth was diluted in $20 \mathrm{~mL}$ of fresh LB broth and grown for $2-3 \mathrm{~h}$ to reach the midexponential growth phase, determined by the growth curve. The bacteria culture was centrifuged for $1 \mathrm{~min}$ at $5000 \mathrm{rpm}$ in $2 \mathrm{~mL}$ centrifuge tubes, and resuspended with physiological saline solution $\left(0.15 \mathrm{M} \mathrm{NaCl}, \mathrm{pH} 7.0,20 \mathrm{mM} \mathrm{NaHCO}_{3}\right.$ ) to the initial optical cell densities at $600 \mathrm{~nm}\left(\mathrm{OD}_{600}\right)$ of $0.15 \pm 0.09$ (the bacterial concentration was $10^{6}-10^{7} \mathrm{CFU} / \mathrm{mL}$, determined by CFU counting method). Circular membrane coupons with $1 \mathrm{~cm}$ in diameter were placed in $15 \mathrm{~mL}$ of sterile plastic tubes with $10 \mathrm{~mL}$ of the bacteria in physiological saline solution and pristine membrane coupons were used as controls. After dynamic incubation for $5 \mathrm{~h}$ with shaking $(200 \mathrm{rpm})$ at $37^{\circ} \mathrm{C}$, the membranes were rinsed gently three times with DI water to remove unattached bacteria. Membrane coupons were then bath-sonicated at $25{ }^{\circ} \mathrm{C}$ for $7 \mathrm{~min}$ in $10 \mathrm{~mL}$ of physiological saline solution to remove the attached bacteria. The obtained suspensions of pristine membrane and DOPA modified membrane were serially diluted 100 times, and $100 \mu \mathrm{L}$ of the bacterial solution of diluted suspension of pristine membrane, DOPA modified membrane and suspension of Ag NPs modified membrane without dilution were plated on LB agar plates, respectively, incubated for $24 \mathrm{~h}$ at $37{ }^{\circ} \mathrm{C}$, and then bacteria colonies were counted. The numbers of live bacteria in the obtained suspension solutions of pristine membranes and Ag NPs modified membranes could be calculated according to the number of colonies and times of dilution, and the percentage of bacterial viability was measured from eq $1 .^{38}$

$$
\text { bacterial viability }=\left(\frac{N_{\mathrm{m}}}{N_{\mathrm{p}}}\right) \times 100 \%
$$

Where $N_{\mathrm{p}}$ and $N_{\mathrm{m}}$ is the numbers of live bacteria in the obtained suspension solutions of pristine membranes and Ag NPs modified membranes, respectively.

2.5. Silver Release from Ag NPs Formed TFC FO Membranes. To test the stability and release behavior of silver from the Ag NPs deposited membranes, the membrane coupon with $1 \mathrm{~cm}$ in radius was 
incubated in $10 \mathrm{~mL}$ of DI water in a small glass vial, and agitated with a shaker. At predetermined time intervals, the solution was removed for ICP-MS analysis, and refilled with $10 \mathrm{~mL}$ of DI water for prolonged release study. For each $10 \mathrm{~mL}$ solution taken out, $0.1 \mathrm{~mL}$ of $68 \%$ nitric acid was added to acidify the solution before ICP-MS analysis. The dissolved silver was quantified with an ICP-MS, and the released silver accumulation was summed upon time. To quantify the $\mathrm{Ag}^{+}$amount in solution, ICP-MS was calibrated by internal standard with ${ }^{115}$ In and a standard curve of ${ }^{107} \mathrm{Ag}$.

2.6. In Situ Regeneration of Ag NPs on the PDA Modified TFC FO Membrane. The Ag NPs deposited membrane coupons were soaked first in a $3.5 \% \mathrm{HNO}_{3}$ solution for $48 \mathrm{~h}$ to deplete the $\mathrm{Ag}$ NPs on the membrane, followed by thorough rinse with deionized water three times. Then, the membrane coupons were placed in 100 $\mathrm{mL}$ of $50 \mathrm{mM}$ silver nitrate aqueous solution and incubated for $24 \mathrm{~h}$ at room temperature to regenerate Ag NPs. The surface morphologies of the Ag NPs regenerated membranes were observed by SEM and the mass loading of regenerated Ag NPs on the membrane was determined by ICP-MS using the above protocol.

2.7. Determination of Water Flux and Salt Flux. The performances of the pristine, PDA modified and in situ generated Ag NPs TFC FO membranes were evaluated using a custom-designed labscale cross-flow forward osmosis test system shown in Figure S1. ${ }^{39}$ The effective surface area of the membrane was $38.52 \mathrm{~cm}^{2}$. The feed solution was DI water and the draw solution was $1 \mathrm{M} \mathrm{NaCl}$ solution. The temperatures of feed and draw solution were keep constant at $25.0 \pm 0.5{ }^{\circ} \mathrm{C}$. The cross-flow rates of both feed and draw solutions were maintained at $0.5 \mathrm{~L} / \mathrm{min}$. The membranes were evaluated under AL-DS (active layer facing draw solution) operational mode. The water flux was calculated as the water weight gain of the draw side per hour and per area of the membrane, and the salt flux was calculated as the salt weight gain of the feed side per hour and per area of the membrane. The water weight gain was monitored by the balance (ME3002, Mettler-Toledo, Switzerland), and the salt weight gain was monitored by the conductivity meter (CON2700, Eutech, USA).

\section{RESULTS AND DISCUSSION}

3.1. Generation of Ag NPs in Situ on Both Sides of PDA Coated TFC FO Membrane. Given that membrane biofouling might occur on both sides of the polyamide active layer and the polysulfone support layer of TFC FO membranes in real applications, ${ }^{14,40}$ incorporation of $\mathrm{Ag}$ NPs on both surfaces is beneficial for enhancing their integral antibiofouling properties. In this study, both surfaces of the TFC FO membranes were involved in the two steps of modification including the PDA coating and the silver nanoparticle generation, as illustrated in Scheme 1.

Scheme 1. In Situ Synthesis Procedure of Silver Nanoparticles on Both Sides of TFC FO Membrane

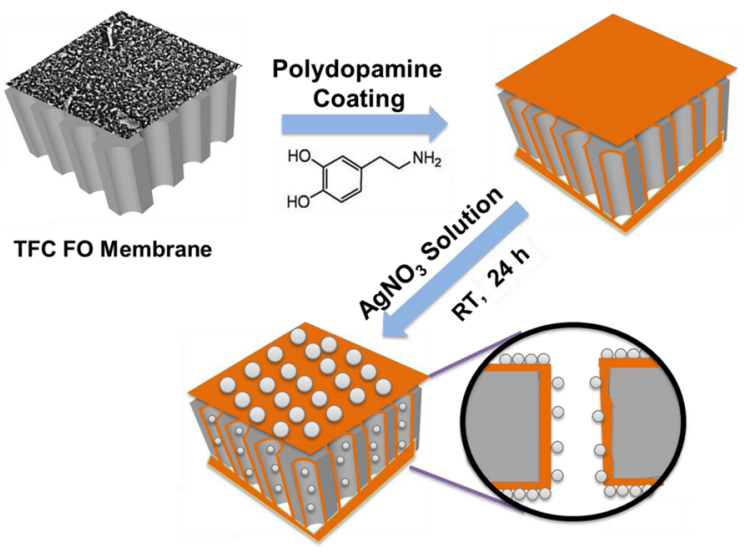

During the first step, the colors of both sides of TFC FO membrane turned from pale to dark, and further to brown with increasing exposure time in dopamine solution of Tris $-\mathrm{HCl}$ buffer ( $\mathrm{pH} 8.5)$, indicating the formation and growth of a PDA layer on membrane surfaces. ${ }^{25}$ Static contact angle measurements were performed on PDA coated polyamide layers to reveal a gradual decrease from $68.4 \pm 1.9^{\circ}$ to $40.6 \pm 4.1^{\circ}$ with prolonging the dopamine polymerization time from 0 to $6 \mathrm{~h}$ (Figure S2), indicating the improved surface hydrophilicity upon the growth of polydopamine. ${ }^{41}$ With prolonging the dopamine polymerization time from 6 to $24 \mathrm{~h}$, the water contact angle of the polyamide surfaces did not drop down further, shown in Figure S2, which may be attributed to the self-inhibition of PDA growth upon $6 \mathrm{~h}$ of dopamine polymerization. ${ }^{27}$ After the second step of incubating the PDA modified membranes with silver nitrate solution, the water contact angle of the polyamide layer was further dropped down to $28.5 \pm 4.6^{\circ}$ upon the formation of Ag NPs (Figure $\mathrm{S} 3 \mathrm{a}$ ), indicating the improved surface hydrophilicity from $\mathrm{Ag}$ NPs. ${ }^{35}$

The surface morphology was observed by scanning electron microscopy (SEM) and atomic force microscopy (AFM). The pristine polyamide surface presents a characteristic uniform ridge-and-valley morphology, shown in Figure 1a, and nonwoven supported polysulfone surface shows a smooth surface, shown in Figure $1 \mathrm{~b}$. After the PDA coating and the incubation with silver salt aqueous solution, dense populated small bright particles with about $20 \mathrm{~nm}$ in average diameter were clearly observed on both surfaces of the membrane, shown in Figure $1 c, d$, and also inside the finger-like pores of polysulfone support, shown in Figure S4. Membrane surface roughness was also characterized and the root-mean-square (RMS) roughness increased from $46.4 \pm 1.6$ to $62.3 \pm 3.7 \mathrm{~nm}$ and to $80.7 \pm 2.2$ $\mathrm{nm}$ for the pristine, PDA coated, and Ag NPs generated polyamide layers of membranes, respectively (Figure S3b,c,d). The polyamide surface obtained a $15.9 \mathrm{~nm}$ increase of the RMS roughness upon the PDA coating, and the generation of $\mathrm{Ag}$ NPs further increased its RMS roughness about $18.4 \mathrm{~nm}$, which is consistent with the size of Ag NPs shown in SEM (Figure 1c).

The surface element composition was examined using energy dispersive X-ray spectroscopy (EDX) and X-ray photoelectron spectroscopy (XPS). EDX confirmed that the small bright particles observed in SEM images were composed of silver (Figure 1e,g) and those regions without nanoparticles had very low level of silver signals (Figure 1f,h), demonstrating silver nanoparticles formed on both sides of TFC FO membranes. The XPS wide scans spectra of the polyamide surfaces, shown in the Figure 2a, and the strong metallic $\mathrm{Ag} 3 \mathrm{~d}$ signal peaks at binding energy of $368 \mathrm{eV}$ (for $\mathrm{Ag} 3 \mathrm{~d}_{5 / 2}$ ) and $374 \mathrm{eV}$ (for $\mathrm{Ag}$ $3 \mathrm{~d}_{3 / 2}$ ), shown in Figure $2 \mathrm{~b}$, further proved the successful generation of Ag NPs on the TFC FO membrane. The atomic percentage of $\mathrm{Ag}$ reached to $1.62 \%$ on the $\mathrm{Ag}$ NPs modified membrane surface (Figure 2c). As a control, the pristine membranes were directly dipped into silver nitrate solution and rinsed with DI water three times under the same conditions as the treatment of PDA-coated membranes to form Ag NPs. The results demonstrated the silver nanoparticles could not form on pristine membrane without PDA layer because no particles were observed by SEM, and no silver signal was detected by EDX (Figure S5). All of these results suggested that the Ag NPs formation was surface independent upon the PDA coating via a 


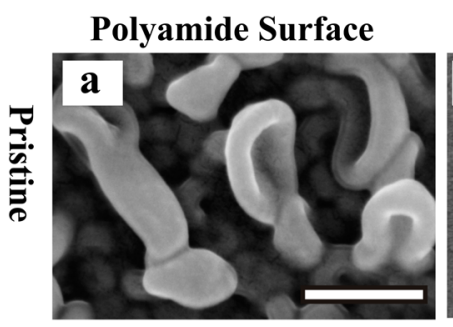

\section{Polysulfone Surface}
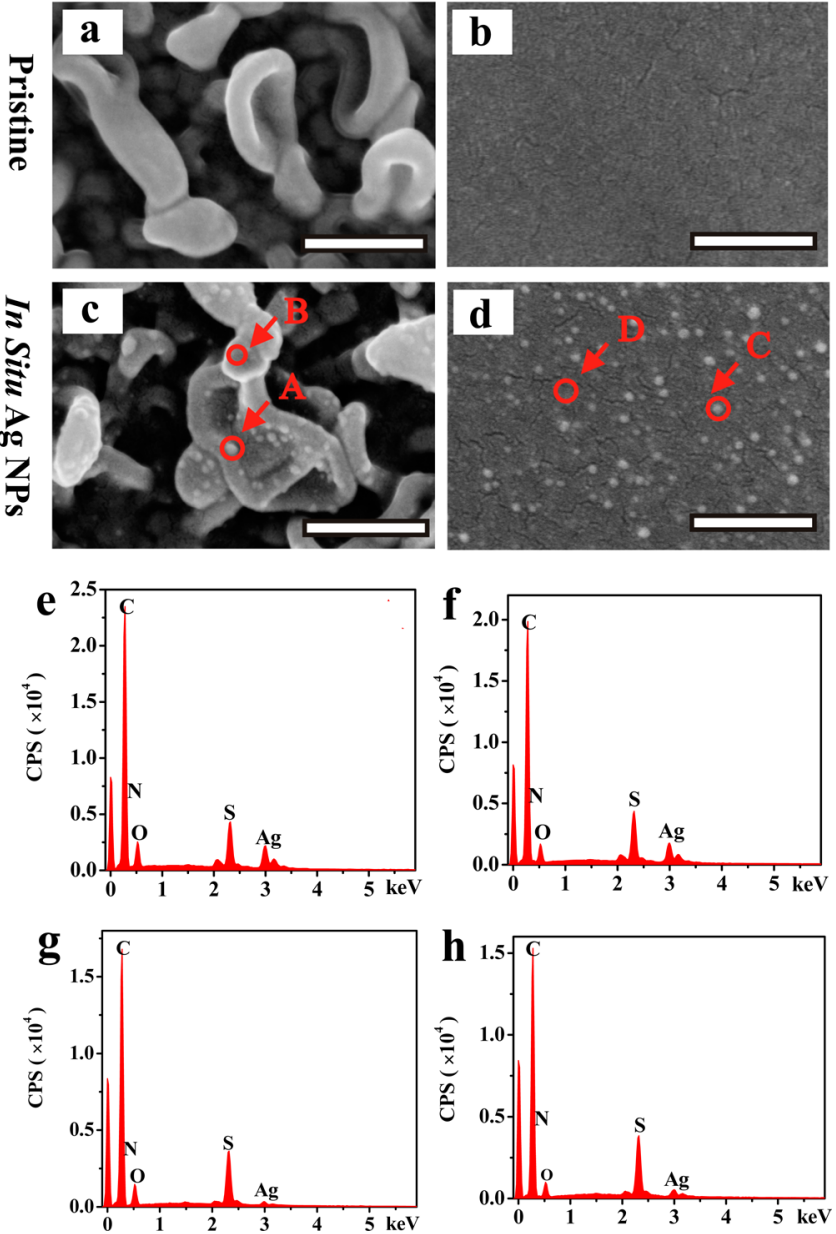

Figure 1. SEM micrographs and EDX spectra of both surfaces of TFC FO membrane before and after Ag NPs deposition: (a and b) polyamide surface and polysulfone support surface of pristine membrane, respectively; ( $c$ and $d$ ) polyamide surface and polysulfone support surface of membrane after in situ generation of Ag NPs; (e, g, $f, h)$ EDX spectra of marked spots A, B in panel $c$ and C and D in panel d, respectively. Scale bar: $200 \mathrm{~nm}$.

simple dip-coating method without any additional reducing agents involved. ${ }^{42-44}$

The mass loadings of Ag NPs were determined by soaking the membrane coupons in $3.5 \% \mathrm{HNO}_{3}$ solution for $48 \mathrm{~h}$ to dissolve the Ag NPs completely. ICP-MS analysis of the dissolved silver solution showed that the silver loadings increased linearly as a function of the dopamine concentration from 0.5 to $10 \mathrm{mg} / \mathrm{mL}$, and the dopamine polymerization time prolonging from $30 \mathrm{~min}$ to $12 \mathrm{~h}$ (Figure $3 \mathrm{a}, \mathrm{b}$ ). Because the increasing dopamine concentration and the prolonging dopamine polymerization time would lead to the growth of the PDA film, ${ }^{25}$ these findings explained the mass loadings of Ag NPs relied on the growth of PDA film and the generation of $\mathrm{Ag}$ NPs occurred not only on the surface of the PDA film but inside the film, consistent with the previous study. ${ }^{34}$

Silver salt concentration was considered to be another critical factor on the Ag NPs mass loading. The population of Ag NPs on the polyamide surfaces increased dramatically with the increasing silver nitrate concentration, shown in SEM images of Figure S6. The mass loading of Ag NPs, determined by ICPMS, increased rapidly from $14.6 \pm 0.9$ to $33.4 \pm 0.3 \mu \mathrm{g} / \mathrm{cm}^{2}$
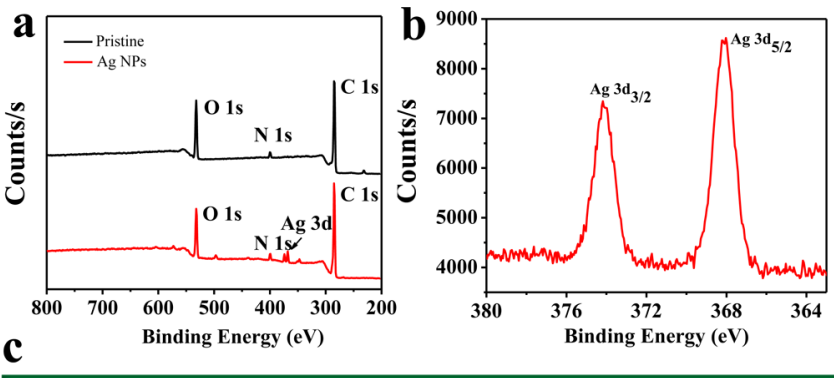

Elemental Compositions (atomic \%)

\begin{tabular}{ccccc} 
Membranes & C 1s & N 1s & O 1s & Ag 3d \\
\cline { 2 - 5 } & & & & \\
\hline Pristine & 76.95 & 2.95 & 20.1 & - \\
Ag NPs & 77.07 & 4.1 & 17.21 & 1.62 \\
\hline
\end{tabular}

Figure 2. XPS wide scans spectra of the polyamide surfaces of pristine (black) and Ag NPs formed (red) TFC FO membrane (a); high resolution XPS spectra of $\mathrm{Ag}$ peak for Ag NPs formed TFC FO membrane in the region between 362 and $382 \mathrm{eV}$ (b); surface elemental compositions of pristine and Ag NPs formed TFC FO membranes obtained from XPS survey scans (c).

with increasing silver nitrate concentration from 10 to $200 \mathrm{mM}$, and then reached a plateau from 200 to $400 \mathrm{mM}$ silver nitrate concentration (Figure 3c). These suggested the generation of silver nanoparticles could reach saturation once the reductive sites were consumed up on the PDA film. Chen et al. also found the loaded silver content in the membrane increased with the prolonged exposure to silver nitrate solution. ${ }^{34}$ Above all, these findings revealed that the Ag NPs mass loading on the membrane could be easily tailored through controlling the growth of PDA film and manipulating the nucleation and generation of silver nanoparticles.

3.2. PDA Coatings Allow Long-Term Silver Release and Rechargeable Ag NPs Generation. The long-term silver release behavior was investigated for one-month simulating sink conditions. The Ag NPs generated TFC FO membrane coupons were immersed in DI water with periodic sampling and DI water refreshing. The released silver content was quantified with ICP-MS after acidification. Figure 4a shows the silver release time profile. In the first 6 days, the cumulated silver amount increased linearly as the release rate of $0.025 \mu \mathrm{g}$ $\mathrm{cm}^{-2} \mathrm{~d}^{-1}$, lower than the reported other Ag NPs immobilized TFC membrane. ${ }^{24}$ Further the silver release rate decreased down to $0.0057 \mu \mathrm{g} \mathrm{cm}^{-2} \mathrm{~d}^{-1}$ from the 6th day to the 26th day. Interestingly, from the $26^{\text {th }}$ day to the $31^{\text {st }}$ day, the silver release rate increased again with $0.018 \mu \mathrm{g} \mathrm{cm}^{-2} \mathrm{~d}^{-1}$ with unknown reason. Compared with the total loading Ag mass as $23.8 \mu \mathrm{g} /$ $\mathrm{cm}^{2}$, the released $\mathrm{Ag}$ mass was $0.38 \mu \mathrm{g} / \mathrm{cm}^{2}$ after 31 days and reached only $1.58 \%$ of the total $\mathrm{Ag}$ amount. This low leaching rate of $0.012 \mu \mathrm{g} \mathrm{cm}^{-2} \mathrm{~d}^{-1}$ is very close to the reported $0.01 \mu \mathrm{g}$ $\mathrm{cm}^{-2} \mathrm{~d}^{-1}$ when Ag NPs were incorporated into the composite membranes through other methods. ${ }^{45}$ The low silver release rate of modified TFC FO membrane indicated that the Ag NPs were immobilized on the membrane surfaces firmly and had a great potential of long lasting antibacterial activities.

SEM was used to observe the surface morphology of the $\mathrm{Ag}$ NPs generated membranes upon silver releasing. The comparable population of the Ag NPs was observed on the 

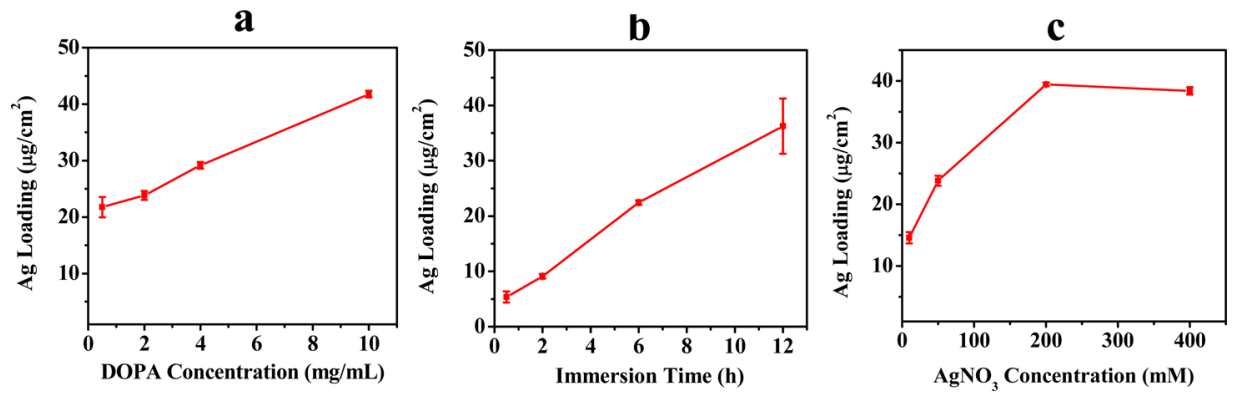

Figure 3. Ag NPs mass loadings on the membranes as a function of dopamine concentration (a), dopamine reaction time (b), and $\mathrm{Ag}^{+}$concentration (c), respectively.
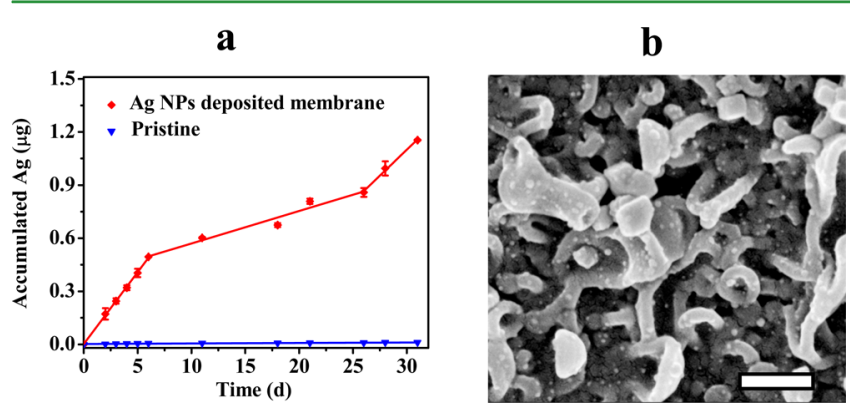

Figure 4. Long-term silver release profile of Ag NPs deposited TFC FO membrane (a), SEM micrograph of Ag NPs TFC FO membrane after 30 days of silver release (b). Scale bar: $200 \mathrm{~nm}$.

freshly prepared Ag NPs membrane (Figure 1c) and on the one released for 31 days (Figure $4 \mathrm{~b}$ ), which confirms the previous release profile with only $1.58 \%$ of $\mathrm{Ag}$ released in the 31 days.

To examine the rechargability of Ag NPs on the PDA coating, the Ag NPs on the membrane coupon were first completely dissolved in $3.5 \% \mathrm{HNO}_{3}$ solution for $48 \mathrm{~h}$, and then regenerated through one step incubation in $50 \mathrm{mM}$ silver nitrate solution for $24 \mathrm{~h}$. The membrane surface color turned light upon the disappearance of Ag NPs and became dark again upon the regeneration of Ag NPs, shown in Figure S7. Small bright spots of Ag NPs disappeared after acidification and $\mathrm{Ag}$ NPs presented after regeneration, shown in SEM images of Figure 5a,b. The ICP-MS results confirmed that the Ag NPs were almost depleted upon acidification with only $0.58 \pm 0.39$ $\mu \mathrm{g} / \mathrm{cm}^{2}$ residual $\mathrm{Ag}$ content and $2.44 \%$ of its original Ag mass loading left, and Ag NPs reformed again upon regeneration with $10.1 \pm 0.5 \mu \mathrm{g} / \mathrm{cm}^{2} \mathrm{Ag}$ mass loading, much higher than the depleted membrane. Even after two cycles of depletion and regeneration, the $\mathrm{Ag}$ loading mass was still high up to $6.5 \pm 0.2$ $\mu \mathrm{g} / \mathrm{cm}^{2}$ on the FO membrane coupon (Figure $5 \mathrm{~d}$ ). Compared with the initial high $\mathrm{Ag}$ NPs loading as $23.8 \mu \mathrm{g} / \mathrm{cm}^{2}$, the regenerated low $\mathrm{Ag}$ NPs loading might be attributed to the decreased reductive sites of the PDA coating soaking in strong acid for a long period of time. ${ }^{26}$ All of these results demonstrate that the Ag NPs can be recharged upon their depletion after the simple dip coating in silver salt solution. It can be concluded that PDA coatings enable a simple and facile approach to recharge Ag NPs for multiple times and thus to keep a longlasting release profile.

3.3. Ag NPs Generated TFC FO Membranes Display Strong Sustainable Antibacterial Properties. The CFU counting method was employed to test the antibacterial properties of Ag NPs modified TFC FO membranes against Gram-negative (E. coli) and Gram-positive (S. aureus) bacteria. The numbers of attached live E. coli decreased by $4.5 \pm 3.6 \%$
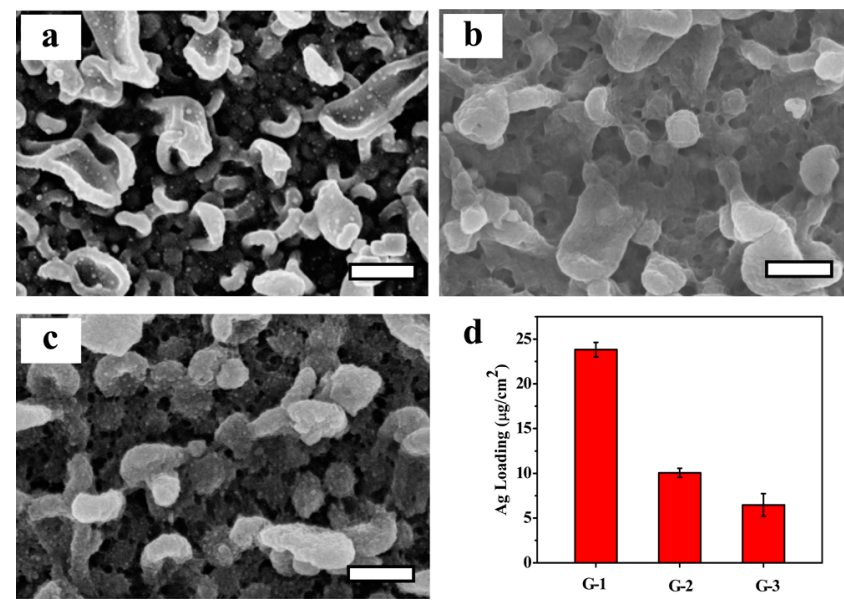

Figure 5. SEM micrographs of the polyamide surface of fresh-prepared Ag NPs TFC FO membranes (a), Ag NPs removal upon treatment with $3.5 \% \mathrm{HNO}_{3}$ solution (b), Ag NPs regeneration upon immersion in $\mathrm{AgNO}_{3}$ solution (c), and the Ag NPs mass loadings of freshprepared Ag NPs TFC FO membrane (G-1), Ag NPs charged membrane for two cycles (G-2), and Ag NPs charged membrane for three cycles (G-3) (d). Scale bar: $200 \mathrm{~nm}$.

and $95.6 \pm 2.4 \%$, respectively, on the PDA modified membrane and the Ag NPs formed membranes, compared with the pristine membrane (Figure 6a). It confirmed that the PDA coating had very little bacterial killing activity against E. coli, and the Ag NPs imparted the FO membrane with an outstanding antibacterial property against E. coli. For S. aureus, the Ag NPs formed FO membrane exhibited excellent antibacterial activities and displayed 4-5 log CFU reduction compared to the pristine membrane. The bacterial viability of Ag NPs formed FO membrane was reduced to $0.01 \%$. The PDA modified membrane showed $37.5 \pm 6.3 \%$ bactericidal efficacy (Figure 6b), which might be attributed to the hydrophilic PDA coating with weak adhesion of $S$. aureus. ${ }^{46}$ The $\mathrm{CFU}$ results also showed that the Ag NPs formed FO membrane still exhibited strong antibacterial activity after leaching of silver for 31 days, with comparable bactericidal efficacy to the freshly prepared $\mathrm{Ag}$ NPs modified membrane (Figure $6 \mathrm{c}$ and Figure S8). In addition, the antibacterial properties of $\mathrm{Ag}$ NPs recharged membranes for a couple of cycles were investigated using CFU methods. Results shown in Figure 6d that all Ag NPs charged membranes for two cycles and three cycles (G-2 and G-3) still exhibited outstanding antibacterial properties against E. coli. The numbers of attached live E. coli decreased by $94.4 \pm 2.3 \%$ and $91.8 \pm 4.3 \%$, respectively on G-2 and G-3 membranes compared with pristine membrane (G-0). Recent studies 

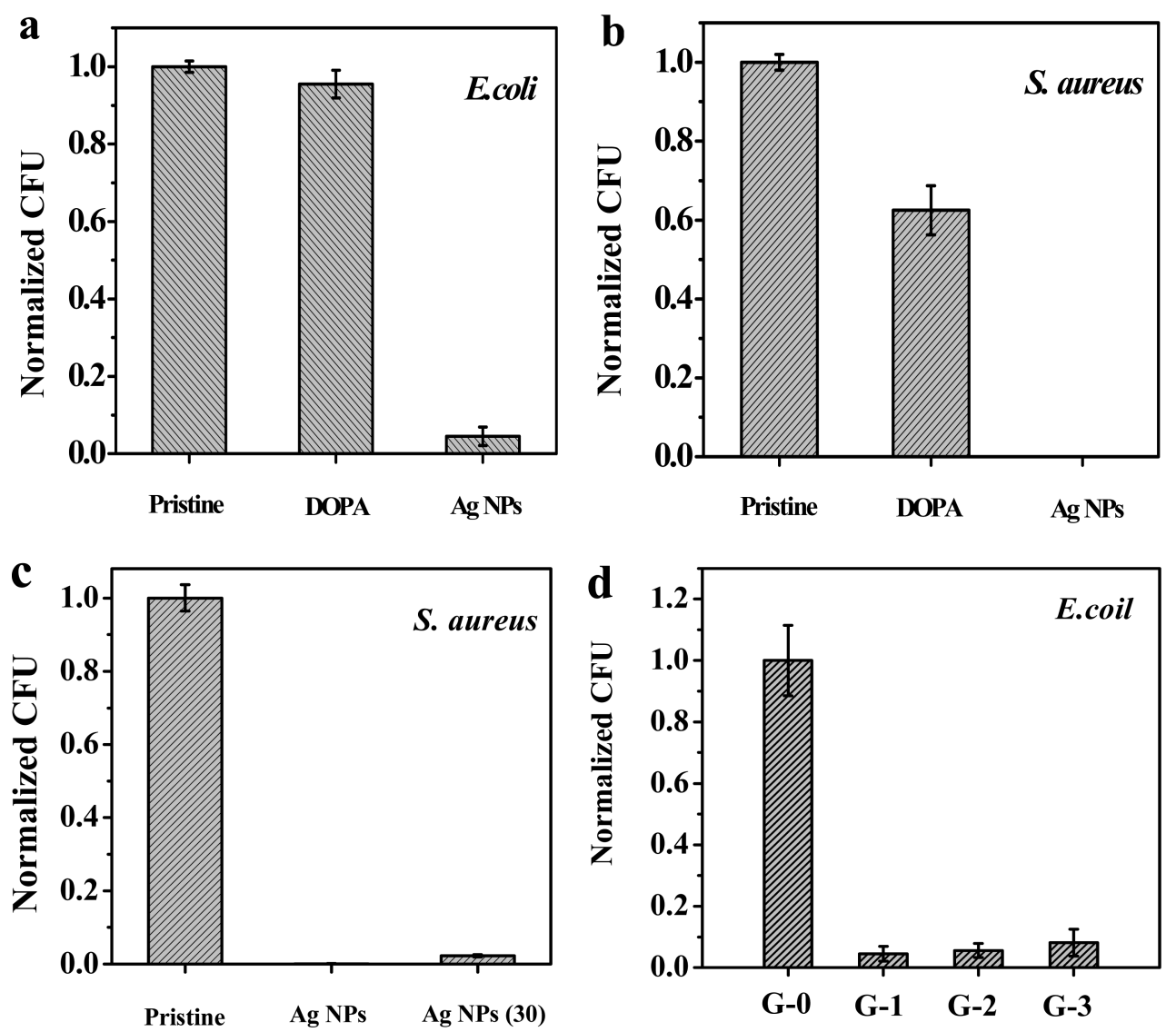

Figure 6. Normalized CFUs of attached live bacteria on membranes after different treatments against E. coli (a) and S. aureus (b), respectively. (c) Normalized CFUs of attached live bacteria on the Ag NPs deposited membrane before and after 30 days of silver release in water (Ag NPs (30)) against S. aureus bacteria. (d) Normalized CFUs of attached live bacteria on the Ag NPs deposited membranes for different cycles against E. coli. G-0, pristine membrane; G-1, Ag NPs deposited membrane for one cycle; G-2, Ag NPs deposited membranes for two cycles; G-3, Ag NPs deposited membranes for three cycles against E. coli. Data are shown as mean \pm SD from three membrane coupons.

demonstrated the antibacterial activity of $\mathrm{Ag}$ NPs worked through free release of silver ions killing mechanism, ${ }^{47,48}$ and the in situ formed Ag NPs on the TFC membrane could work as an immediate source of silver ions to suppress subsequent proliferation of bacteria effectively. By considering the longterm release profile and rechargability of the Ag NPs, we expect that our Ag NPs generation based on the PDA coating is sustainable for long period and is persistent for strong antibacterial activity to mitigate membrane biofouling.

3.4. Ag NPs has Controllable Effect on the Water Flux and Salt Permeability of TFC FO Membranes. The water flux and salt permeability of the membranes were tested using a custom lab-scale cross-flow forward osmosis test system in ALDS membrane orientation. Figure 7 shows the water flux decreased from $17.49 \pm 0.42$ to $14.56 \pm 0.75 \mathrm{~L} \mathrm{~m}^{-2} \mathrm{~h}^{-1}$ and $13.31 \pm 0.95 \mathrm{~L} \mathrm{~m}^{-2} \mathrm{~h}^{-1}$, and the reverse salt flux decreased from $34.64 \pm 2.6$ to $28.24 \pm 3.61 \mathrm{~g} \mathrm{~m}^{-2} \mathrm{~h}^{-1}$ and $22.39 \pm 0.54 \mathrm{~g}$ $\mathrm{m}^{-2} \mathrm{~h}^{-1}$, for pristine, PDA coated, and Ag NPs generated membranes, respectively. The PDA coating decreased the water flux and the reverse salt flux by $16.75 \%$ and $18.48 \%$, and the $\mathrm{Ag}$ NPs generation decreased the water flux and the reverse salt flux further by $8.58 \%$ and $20.71 \%$. Therefore, the PDA coatings had stronger effect on decreasing the water flux than the $\mathrm{Ag}$ NPs generation, whereas the Ag NPs generation had more benefits on suppressing the reverse salt flux than the PDA coatings. We also demonstrated the water flux and reverse salt flux could be tailored via controlling the formation of PDA

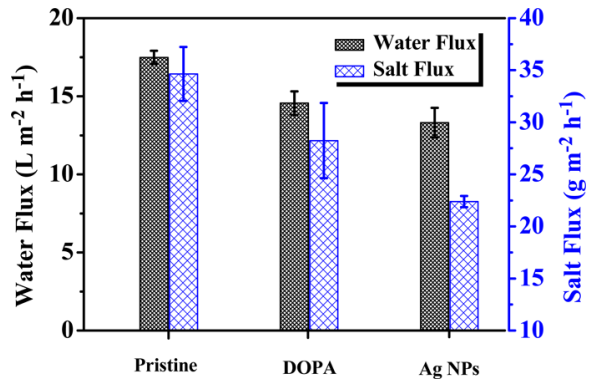

Figure 7. Water flux and reverse salt flux of TFC FO membranes tested in the AL-DS operational mode. Results were an average of three different membrane coupons. Error bars are given as standard deviation. The draw solution and feed solution were $1 \mathrm{M} \mathrm{NaCl}$ solution and deionized water, respectively. The FO test ran at $25^{\circ} \mathrm{C} \pm$ $0.1{ }^{\circ} \mathrm{C}$ with $0.5 \mathrm{~L} / \mathrm{min}$ of both cross-flow velocities of feed and draw solution.

coating by adjusting the immersion time of membranes in DOPA solution (Figure S9). Our current study suggests that the water flux and the reverse salt flux of the TFC FO membranes can be further optimized to achieve controllable effect through optimizing the thickness of the PDA film and the growth of Ag NPs. 


\section{CONCLUSIONS}

In this study, a simple and facile approach has been developed to achieve strong sustainable antibacterial properties on the TFC FO membrane. We have demonstrated that the Ag NPs could form on both sides of TFC FO membranes upon the synthesis of PDA film, and had a long-term silver release profile with rechargability for multiple times upon their depletion. The Ag NPs deposited membranes exhibited strong sustainable bacterial killing activities against Gram-negative bacteria and Gram-positive bacteria, and had controllable effect on the membrane performances including the water flux and reverse salt flux in the FO test mode. Thus, such an approach provides a practicable avenue for mitigating long-term membrane biofouling of the FO membrane. Because the PDA coating can form on various substrates including metals, plastics, and other materials, ${ }^{25} \mathrm{Ag}$ NPs generation would be expected to be surface-independent once PDA coating formed. Our practicable antibacterial strategy may apply to other types of filtration membranes with diverse material surfaces and may pave a new way to achieve the sustainable membrane antibiofouling on a large scale.

\section{ASSOCIATED CONTENT}

\section{(S) Supporting Information}

The Supporting Information is available free of charge on the ACS Publications website at DOI: 10.1021/acsami.6b06727.

Schematic drawing of the custom-designed lab-scale cross-flow forward osmosis test system (Figure S1); water contact angles of the TFC FO membrane changing with immersion time in dopamine solution (Figure S2); water contact angels (a) and AFM analyses of pristine, polydopamine modified and in situ generated Ag NPs TFC FO membranes (b-d) (Figure S3); SEM micrographs of cross sections of FO membrane after in situ generation of Ag NPs at different magnifications (Figure S4); surface morphology and elemental analysis of polyamide layer of pristine membrane coupon directly dipped into silver nitrate solution and rinsed with water (Figure S5); SEM micrographs of polyamide layer of $\mathrm{Ag}$ NPs modified membranes with the concentration of silver nitrate aqueous solution increasing from $10 \mathrm{mM}$ to $200 \mathrm{mM}$ (Figure S6); photographs of Ag NPs formed membranes and after treatment with $3.5 \% \quad \mathrm{HNO}_{3}$ solution for $48 \mathrm{~h}$ and $\mathrm{Ag}$ NPs regenerated membranes via immersion in $\mathrm{AgNO}_{3}$ solution again for $24 \mathrm{~h}$ (Figure S7); antimicrobial activities photographs of each membrane against $S$. aureus (Figure S8); normalized water flux and reverse salt flux of DOPA modified TFC FO membranes with different immersion time (Figure S9) (PDF).

\section{AUTHOR INFORMATION}

\section{Corresponding Author}

*Y. Hu. Tel: +86-535-2109236. E-mail: yunxiahu@yic.ac.cn.

\section{Notes}

The authors declare no competing financial interest.

\section{ACKNOWLEDGMENTS}

The authors gratefully acknowledge the advises from Professor Menachem Elimelech (Department of Chemical and Environmental Engineering, Yale University, New Haven), and the funding support from National Natural Science Foundation of China (No. 21476249), and Key Science and Technology Program of Shandong Province (No. 2014GHY115021).

\section{REFERENCES}

(1) Elimelech, M.; Phillip, W. A. The Future of Seawater Desalination: Energy, Technology, and the Environment. Science 2011, 333 (6043), 712-717.

(2) Fane, A. G.; Wang, R.; Hu, M. X. Synthetic Membranes for Water Purification: Status and Future. Angew. Chem., Int. Ed. 2015, 54 (11), 3368-3386.

(3) Duong, P. H.; Chung, T.-S. Application of Thin Film Composite Membranes with Forward Osmosis Technology for the Separation of Emulsified Oil-Water. J. Membr. Sci. 2014, 452, 117-126.

(4) Cath, T. Y.; Childress, A. E.; Elimelech, M. Forward Osmosis: Principles, Applications, and Recent Developments. J. Membr. Sci. 2006, 281 (1), 70-87.

(5) Hickenbottom, K. L.; Hancock, N. T.; Hutchings, N. R.; Appleton, E. W.; Beaudry, E. G.; Xu, P.; Cath, T. Y. Forward Osmosis Treatment of Drilling Mud and Fracturing Wastewater From Oil and Gas Operations. Desalination 2013, 312, 60-66.

(6) Coday, B. D.; Xu, P.; Beaudry, E. G.; Herron, J.; Lampi, K.; Hancock, N. T.; Cath, T. Y. The Sweet Spot of Forward Osmosis: Treatment of Produced Water, Drilling Wastewater, and Other Complex and Difficult Liquid Streams. Desalination 2014, 333 (1), 23-35.

(7) Rastogi, N. K. Opportunities and Challenges in Application of Forward Osmosis in Food Processing. Crit. Rev. Food Sci. Nutr. 2016, 56 (2), 266-291.

(8) Logan, B. E.; Elimelech, M. Membrane-Based Processes for Sustainable Power Generation Using Water. Nature 2012, 488 (7411), 313-319.

(9) Yip, N. Y.; Tiraferri, A.; Phillip, W. A.; Schiffman, J. D.; Hoover, L. A.; Kim, Y. C.; Elimelech, M. Thin-Film Composite Pressure Retarded Osmosis Membranes for Sustainable Power Generation From Salinity Gradients. Environ. Sci. Technol. 2011, 45 (10), 43604369.

(10) Zhao, S.; Zou, L.; Tang, C. Y.; Mulcahy, D. Recent Developments in Forward Osmosis: Opportunities and Challenges. J. Membr. Sci. 2012, 396, 1-21.

(11) Bar-Zeev, E.; Perreault, F.; Straub, A. P.; Elimelech, M. Impaired Performance of Pressure-Retarded Osmosis Due to Irreversible Biofouling. Environ. Sci. Technol. 2015, 49 (21), 13050-13058.

(12) Kwan, S. E.; Bar-Zeev, E.; Elimelech, M. Biofouling in Forward Osmosis and Reverse Osmosis: Measurements and Mechanisms. J. Membr. Sci. 2015, 493, 703-708.

(13) Gao, W.; Liang, H.; Ma, J.; Han, M.; Chen, Z.-l.; Han, Z.-s.; Li, G.-b. Membrane Fouling Control in Ultrafiltration Technology for Drinking Water Production: a Review. Desalination 2011, 272 (1), 18.

(14) Yoon, H.; Baek, Y.; Yu, J.; Yoon, J. Biofouling Occurrence Process and Its Control in the Forward Osmosis. Desalination 2013, $325,30-36$.

(15) Chernousova, S.; Epple, M. Silver as Antibacterial Agent: Ion, Nanoparticle, and Metal. Angew. Chem., Int. Ed. 2013, 52 (6), 16361653.

(16) Le Ouay, B.; Stellacci, F. Antibacterial Activity of Silver Nanoparticles: A Surface Science Insight. Nano Today 2015, 10 (3), 339-354.

(17) Madhavan, P.; Hong, P.-Y.; Sougrat, R.; Nunes, S. P. SilverEnhanced Block Copolymer Membranes with Biocidal Activity. ACS Appl. Mater. Interfaces 2014, 6 (21), 18497-18501.

(18) Andrade, P. F.; de Faria, A. F.; Oliveira, S. R.; Arruda, M. A. Z.; do Carmo Gonçalves, M. Improved Antibacterial Activity of Nanofiltration Polysulfone Membranes Modified with Silver Nanoparticles. Water Res. 2015, 81, 333-342.

(19) Li, X.; Pang, R.; Li, J.; Sun, X.; Shen, J.; Han, W.; Wang, L. In Situ Formation of $\mathrm{Ag}$ Nanoparticles in PVDF Ultrafiltration 
Membrane to Mitigate Organic and Bacterial Fouling. Desalination 2013, 324, 48-56.

(20) Destaye, A. G.; Lin, C.-K.; Lee, C.-K. Glutaraldehyde Vapor Cross-Linked Nanofibrous PVA Mat with in Situ Formed Silver Nanoparticles. ACS Appl. Mater. Interfaces 2013, 5 (11), 4745-4752. (21) Ben-Sasson, M.; Lu, X.; Bar-Zeev, E.; Zodrow, K. R.; Nejati, S.; Qi, G.; Giannelis, E. P.; Elimelech, M. In Situ Formation of Silver Nanoparticles on Thin-Film Composite Reverse Osmosis Membranes for Biofouling Mitigation. Water Res. 2014, 62, 260-270.

(22) Li, J.-H.; Shao, X.-S.; Zhou, Q.; Li, M.-Z.; Zhang, Q.-Q. The Double Effects of Silver Nanoparticles on the PVDF Membrane: Surface Hydrophilicity and Antifouling Performance. Appl. Surf. Sci. 2013, 265, 663-670.

(23) Park, S. H.; Ko, Y. S.; Park, S. J.; Lee, J. S.; Cho, J.; Baek, K. Y.; Kim, I. T.; Woo, K.; Lee, J. H. Immobilization of Silver NanoparticleDecorated Silica Particles on Polyamide Thin Film Composite Membranes for Antibacterial Properties. J. Membr. Sci. 2016, 499, 80-91.

(24) Yin, J.; Yang, Y.; Hu, Z.; Deng, B. Attachment of Silver Nanoparticles (AgNPs) onto Thin-Film Composite (TFC) Membranes Through Covalent Bonding to Reduce Membrane Biofouling. J. Membr. Sci. 2013, 441, 73-82.

(25) Lee, H.; Dellatore, S. M.; Miller, W. M.; Messersmith, P. B. Mussel-Inspired Surface Chemistry for Multifunctional Coatings. Science 2007, 318 (5849), 426-430.

(26) Liu, Y.; Ai, K.; Lu, L. Polydopamine and Its Derivative Materials: Synthesis and Promising Applications in Energy, Environmental, and Biomedical Fields. Chem. Rev. 2014, 114 (9), 5057-5115.

(27) Jiang, J.; Zhu, L.; Zhu, L.; Zhang, H.; Zhu, B.; Xu, Y. Antifouling and Antimicrobial Polymer Membranes Based on Bioinspired Polydopamine and Strong Hydrogen-Bonded Poly (N-vinyl pyrrolidone). ACS Appl. Mater. Interfaces 2013, 5 (24), 12895-12904.

(28) Li, Y.; Su, Y.; Zhao, X.; He, X.; Zhang, R.; Zhao, J.; Fan, X.; Jiang, Z. Antifouling, High-Flux Nanofiltration Membranes Enabled by Dual Functional Polydopamine. ACS Appl. Mater. Interfaces 2014, 6 (8), 5548-5557.

(29) Yang, H.-C.; Luo, J.; Lv, Y.; Shen, P.; Xu, Z.-K. Surface Engineering of Polymer Membranes via Mussel-Inspired Chemistry. J. Membr. Sci. 2015, 483, 42-59.

(30) Cong, Y.; Xia, T.; Zou, M.; Li, Z.; Peng, B.; Guo, D.; Deng, Z. Mussel-Inspired Polydopamine Coating as a Versatile Platform for Synthesizing Polystyrene/Ag Nanocomposite Particles with Enhanced Antibacterial Activities. J. Mater. Chem. B 2014, 2 (22), 3450-3461.

(31) Guo, L.; Liu, Q.; Li, G.; Shi, J.; Liu, J.; Wang, T.; Jiang, G. A mussel-Inspired Polydopamine Coating as a Versatile Platform for the in Situ Synthesis of Graphene-Based Nanocomposites. Nanoscale 2012, 4 (19), 5864-5867.

(32) Lu, Z.; Xiao, J.; Wang, Y.; Meng, M. In Situ Synthesis of Silver Nanoparticles Uniformly Distributed on Polydopamine-Coated Silk Fibers for Antibacterial Application. J. Colloid Interface Sci. 2015, 452, $8-14$.

(33) Sileika, T. S.; Kim, H.-D.; Maniak, P.; Messersmith, P. B. Antibacterial Performance of Polydopamine-Modified Polymer Surfaces Containing Passive and Active Components. ACS Appl. Mater. Interfaces 2011, 3 (12), 4602-4610.

(34) Tang, L.; Livi, K. J.; Chen, K. L. Polysulfone Membranes Modified with Bioinspired Polydopamine and Silver Nanoparticles Formed in Situ To Mitigate Biofouling. Environ. Sci. Technol. Lett. 2015, 2 (3), 59-65.

(35) Huang, L.; Zhao, S.; Wang, Z.; Wu, J.; Wang, J.; Wang, S. In Situ Immobilization of Silver Nanoparticles for Improving Permeability, Antifouling and Anti-Bacterial Properties of Ultrafiltration Membrane. J. Membr. Sci. 2016, 499, 269-281.

(36) Li, X.; Zhang, S.; Fu, F.; Chung, T.-S. Deformation and Reinforcement of Thin-Film Composite (TFC) Polyamide-Imide (PAI) Membranes for Osmotic Power Generation. J. Membr. Sci. 2013, 434, 204-217.

(37) Sutton, S. Accuracy of Plate Counts. J. Valid. Technol. 2011, 17

(3), $42-46$.
(38) Park, S. H.; Kim, S. H.; Park, S. J.; Ryoo, S.; Woo, K.; Lee, J. S.; Kim, T. S.; Park, H. D.; Park, H.; Park, Y. I.; Cho, J.; Lee, J. H. Direct Incorporation of Silver Nanoparticles onto Thin-Film Composite Membranes via Arc Plasma Deposition for Enhanced Antibacterial and Permeation Performance. J. Membr. Sci. 2016, 513, 226-235.

(39) Mi, B.; Elimelech, M. Chemical and Physical Aspects of Organic Fouling of Forward Osmosis Membranes. J. Membr. Sci. 2008, 320 (1), 292-302.

(40) Lu, X.; Arias Chavez, L. H.; Romero-Vargas Castrillón, S.; Ma, J.; Elimelech, M. Influence of Active Layer and Support Layer Surface Structures on Organic Fouling Propensity of Thin-Film Composite Forward Osmosis Membranes. Environ. Sci. Technol. 2015, 49 (3), $1436-1444$.

(41) Huang, L.; Arena, J. T.; Manickam, S. S.; Jiang, X.; Willis, B. G.; McCutcheon, J. R. Improved Mechanical Properties and Hydrophilicity of Electrospun Nanofiber Membranes for Filtration Applications by Dopamine Modification. J. Membr. Sci. 2014, 460, 241-249.

(42) Wang, B.; Liu, Y.; Zhang, Y.; Guo, Z.; Zhang, H.; Xin, J. H.; Zhang, L. Bioinspired Superhydrophobic $\mathrm{Fe}_{3} \mathrm{O}_{4} @$ Polydopamine@Ag Hybrid Nanoparticles for Liquid Marble and Oil Spill. Adv. Mater. Interfaces 2015, 2 (13), 1500234.

(43) Zhang, Z.; Zhang, J.; Zhang, B.; Tang, J. Mussel-Inspired Functionalization of Graphene for Synthesizing Ag-PolydopamineGraphene Nanosheets as Antibacterial Materials. Nanoscale 2013, 5 (1), 118-123.

(44) Jeon, E. K.; Seo, E.; Lee, E.; Lee, W.; Um, M.-K.; Kim, B.-S. Mussel-Inspired Green Synthesis of Silver Nanoparticles on Graphene Oxide Nanosheets for Enhanced Catalytic Applications. Chem. Commun. 2013, 49 (33), 3392-3394.

(45) Liu, S.; Zhang, M.; Fang, F.; Cui, L.; Wu, J.; Field, R.; Zhang, K. Biogenic Silver Nanocomposite TFC Nanofiltration Membrane with Antifouling Properties. Desalin. Water Treat. 2016, 57, 10560-10571.

(46) Karkhanechi, H.; Takagi, R.; Matsuyama, H. Biofouling Resistance of Reverse Osmosis Membrane Modified with Polydopamine. Desalination 2014, 336, 87-96.

(47) Rizzello, L.; Pompa, P. P. Nanosilver-Based Antibacterial Drugs and Devices: Mechanisms, Methodological Drawbacks, and Guidelines. Chem. Soc. Rev. 2014, 43 (5), 1501-1518.

(48) Xiu, Z.; Zhang, Q.; Puppala, H.; Colvin, V.; Alvarez, P. J. J. Negligible Particle-Specific Antibacterial Activity of Silver Nanoparticles. Nano Lett. 2012, 12 (8), 4271-4275. 